British Journal of Psychiatry (1987), 150, 557-571

\title{
Correspondence
}

Editor: Greg Wilkinson

\section{Consensus Statement: Panic Disorder}

SIR: We wish to draw the attention of readers to a recent initiative concerning the topic of panic. In November 1986 an informal meeting was held in the UK to discuss anxiety and panic, with particular reference to diagnosis and classification. The participants, listed below, came from several academic and clinical centres in the UK and represented a wide range of expertise and specialist interests within this area of psychiatry. Various topics were outlined in order to focus the subsequent discussions.

The concept of anxiety neurosis was established nearly 100 years ago, and has emphasised at several times since the somatic, particularly cardiovascular, symptoms. 'Panic' originally implied collective and excessive fear reactions, but the term has come to have somewhat different psychiatric implications, stressing individual physiological response. Cardinal features are the rapid rate of onset of the attack and the feeling of loss of personal control. Recently, attempts have been made to separate panic and anxiety as diagnostic entities, both in the proposed ICD-10 and in the DSM-III classification system of the American Psychiatric Association.

The epidemiology of panic attacks and disorders varies according to the sample studied and the criteria used: population studies, surveys of general practice attenders, and out-patient studies provide very different prevalences of panic. In one American study, prevalences were: Generalised Anxiety Disorder 2.5\%; Phobic Disorders 1.5\%; and Panic Disorders $0.5 \%$ of the general population. One study suggests a low use of psychiatric services; others, a higher use of general practice services. Distinct diagnostic categories probably give arbitrary estimates of prevalence. An approach regarding severity of panic attacks as an important dimension gives more realistic estimates: for example, in a recent UK study occasional panics were present in 1 in 10 , whereas frequent disabling panic attacks were reported by only 1 in 50 of the adult population. Alcohol can be involved in a vicious circle of panic leading to problem drinking leading to withdrawal leading to panic, although this is less common in the community than in out-patient psychiatric practice. The natural history of panic either as a cluster of symptoms occurring alone or forming part of another psychiatric illness has not been established.
Family studies show that anxiety neuroses are familial, and the data from twin studies suggest a genetic contribution. This is more obvious in Panic than in Generalised Anxiety Disorder, and this has led some to suggest a specific genetic contribution to the former but not the latter. However, more general genetic predispositions are likley to be important: personality, neuroticism, autonomic lability, and biochemical factors. Multifactorial or polygenic explanations may, therefore, fit the data better.

In the study of anxiety and panic, reliable measures are important, and self-reports must remain a key feature; several dimensions should be taken into account. These include severity, duration, and frequency of attacks as well as type, e.g. 'spontaneous' as opposed to 'situation-related' panic attacks. The diary method is very useful. Intensity is best assessed by the disruptive effects of the attack on normal on-going activities. Other aspects of the psychiatric condition should be rated, such as phobias and levels of generalised anxiety and depression. The natural history of the panic attacks should be documented, for example whether the attacks follow an episodic, cyclic, or clustered pattern.

Views on the psychological aspects of panic attacks currently focus on the cognitive hypothesis. This proposes a cycle in which a trigger stimulus, internal or external, gives rise to perceived threat, apprehension, and in particular to bodily sensations which are misinterpreted in a catastrophic fashion and thereby reinforce the perceived threat. These misinterpretations involve perceiving these sensations as indicating immediate and severe physical or mental harm. In cognitive therapy these misinterpreted bodily sensations are identified, and by suggesting and testing alternative non-catastrophic interpretations panic and anxiety levels are reduced. There is some evidence, stemming from studies on cognitive abnormalities, that panic can be differentiated from generalised anxiety disorders.

The bulk of morbidity in anxiety disorders is encountered in general practice, but there are problems with current diagnostic schemes. Patients may present with anxiety, phobic, panic, and depressive symptoms in various combinations. As symptomclusters are not stable over time, there are often changes in appropriate diagnostic labels; somatisation is common, so that patients who experience 
panic attacks often complain of bodily rather than psychological symptoms. This may lead either to failure of recognition or inappropriate referral.

The treatment of panic disorders is varied. Most of the evidence comes from studies which included agoraphobic patients with panic attacks, and concurrent behavioural treatments were usually given. Tricyclic antidepressants, such as imipramine and clomipramine, have an established place in the prevention of panic attacks. Where panic occurs without significant depression some workers have evidence to support the use of low doses of antidepressant drugs, but others have suggested the use of high doses. There is also disagreement about the time of onset of the therapeutic effect, some workers reporting prompt onset, others noting substantial delays. Some panic patients appear very sensitive to unwanted effects of these drugs. Reponse at low doses may suggest a mechanism of action distinct from the antidepressant action seen at higher doses. Although improvement may be maintained for some time after stopping the drugs, over the longer term the outcome is more uncertain. A few benzodiazepines used in doses above the usual anxiolytic ones (e.g. alprazolam, clonazepam, diazepam) have been shown to prevent panic attacks; the evidence for this is most clear for alprazolam, and further studies are in progress. Such response to benzodiazepines is, however, less convincing than that to the tricyclic antidepressants, and there is a danger of dependence. Alternative drugs include the MAOIs and $\beta$ adrenoceptor blockers; the latter are of limited usefulness. All of these treatments can be used in conjunction with behavioural and cognitive therapies. Indeed, preliminary evidence suggests the efficacy of cognitive therapy alone in patients with panics. High consultation rates and limited time in general practice emphasise the need for simple, pragmatic therapies.

Panic attacks can be regarded as a cluster of symptoms which can occur alone or as part of another disorder which has its own place in the classificatory scheme. Controversy attends the nosological status of disorders in which the symptoms of panic occur in conjunction with other disorders (agoraphobia, generalised anxiety, or depression). In the American classification (Revised DSM-III) panic attacks are given primacy and are thought to delineate a separate diagnostic entity (called Panic Disorder). The proposed revision of the ICD uses the term 'Panic Disorder (episodic anxiety)' to describe a disorder in which panic attacks occur as the major feature in the absence of significant anxiety between attacks and a depressive disorder. The meeting preferred the latter convention, as it is unclear whether panic attacks which occur in association with significant generalised anxiety are best considered as a separate disorder or as a more severe form of generalised anxiety disorder.

The meeting also recognised that for research purposes the individual symptom clusters should be considered independently, and it urged longitudinal studies to clarify their natural history and outcome.

The consensus of the meeting was that it is useful descriptively to recognise the symptom cluster of panic attacks which can be defined operationally in terms of features and content. Frequency is a useful, albeit arbitrary, indicator of severity. It was recognised that patients are encountered in whom panic attacks occur in the absence of other significant symptoms. The status of panic disorder as a separate entity was not strongly supported by available clinical and scientific evidence. Finally, although some evidence suggested that panic attacks often represented a severe form of more generalised anxiety, it was premature to attempt to describe the relationships between the various symptom clusters.

Participants: G. W. AshCRofT (Aberdeen); G. BEAUMONT (Stockport); J. BONN (London); S. BrandoN (Leicester); A. BRIGGS (Leicester); D. Clark (Oxford); K. Davison (Newcastle-uponTyne); M. G. Gelder (Oxford); D. GoldBerg (Manchester); R. HerRINGTON (Glasgow); M. C. KHAN (Hartlepool); M. LADER, Chairman (London); M. S. LiPSEDGE (London); A. MACDONALD (London); P. MAguIRE (Manchester); P. T. S. MILLN (Southampton); R. M. MURRAY (London); R. F. Stirton (Leicester); A. C. P. Sims (Leeds); R. P. SNaIth (Leeds); D. Wheatley (Hinchley Wood).

(See also pp 563-564)

\section{Senile Dementia and Parietal Lobe Dysfunction}

SIR: Gilleard et al (Journal, January 1987, 150, 114-117) miss the point of the paper by McDonald (1969), whose findings they claim to have disproved. Since the latter's hypothesis was essentially a predictive one which was vindicated by follow-up and confirmed by others (Naguib \& Levy, 1982) it is difficult to see how a purely cross-sectional study with no follow-up can be strictly relevant to the issue. Furthermore, Gilleard et al claim that McDonald did not exclude cases suggestive of a vascular aetiology, whereas the 1969 paper states that such exclusion did occur: "If there was a history of strokes or epileptic seizures or if there were focal neurological signs the patients were excluded as being suspect arteriosclerotic dementias". 